\title{
O-antigen expression in Salmonella enterica serovar Typhi is regulated by nitrogen availability through RpoN-mediated transcriptional control of the rfaH gene
}

\author{
Mauricio Bittner, ${ }^{1}$ Soledad Saldías, ${ }^{1}$ Claudia Estévez, ${ }^{1}$ Mercedes Zaldívar, ${ }^{1}$ \\ Cristina L. Marolda, ${ }^{2}$ Miguel A. Valvano ${ }^{2}$ and Inés Contreras ${ }^{1}$
}

Author for correspondence: Inés Contreras. Tel: +562678 1658. Fax: +5622227900. e-mail: inescon@ciq.uchile.cl

1 Departamento de Bioquímica y Biología Molecular, Facultad de Ciencias Químicas y Farmacéuticas, Universidad de Chile, PO Box 174, Correo 22, Santiago, Chile

2 Department of Microbiology and Immunology, University of Western Ontario, London, Ontario, Canada N6A 5C1
The authors previously reported increased expression of the Salmonella enterica serovar Typhi (S. typhi) rfaH gene when the bacterial cells reach stationary phase. In this study, using a lacz fusion to the $r f a H$ promoter region, they demonstrate that growth-dependent regulation of $r f a H$ expression occurs at the level of transcription initiation. It was also observed that production of the lipopolysaccharide (LPS) 0-antigen by S. typhi Ty2 correlated with the differential expression of $r f a H$ during bacterial growth. This was probably due to the increased cellular levels of $\mathrm{RfaH}$, since expression of the distal gene in the O-antigen gene cluster of S. typhi Ty2, wbaP, was also increased during stationary growth, as demonstrated by RT-PCR analysis. Examination of the sequences upstream of the $r f a H$ coding region revealed homologies to potential binding sites for the RcsB/RcsA dimer of the RcsC/YopJ/RcsB phosphorelay regulatory system and for the RpoN alternative sigma factor. The expression of the rfaH gene in rpoN and rcsB mutants of $S$. typhi Ty2 was measured. The results indicate that inactivation of $r p o N$, but not of $\operatorname{rcs} B$, suppresses the growth-phase-dependent induction of $r f a H$ expression. Furthermore, production of $\beta$-galactosidase mediated by the $r f a H-l a c z$ fusion increased approximately fourfold when bacteria were grown in a nitrogenlimited medium. Nitrogen limitation was also shown to increase the expression of the 0 -antigen by the wild-type $S$. typhi Ty2, as demonstrated by a similar electrophoretic profile to that observed during the stationary phase of growth in rich media. It is therefore concluded that the relationship between LPS production and nitrogen limitation parallels the pattern of $r f a H$ regulation under the control of RpoN and is consistent with the idea that RpoN modulates LPS formation via its effect on $\mathrm{rfaH}$ gene expression during bacterial growth.

Keywords: lipopolysaccharide, regulation, transcription, sigma factor

\section{INTRODUCTION}

Salmonella enterica serovar Typhi (herein abbreviated as $S$. typhi) causes typhoid fever in humans, a disease of great public health concern in many developing countries (Pang et al., 1998). The detailed molecular mechanisms specifically involved in the pathogenesis of S. typhi are poorly understood due to the lack of an animal model. However, a great deal of information has been obtained from studies using S. enterica serovar Typhimurium, which infects a wide spectrum of animal hosts and causes a typhoid-like disease in the mouse (Jones \& Falkow, 1996). We are interested in the analysis of the biosynthesis and regulation of S. typhi surface polysaccharides, especially the lipopolysaccharide (LPS). This glycolipid surface molecule is an abundant component of the bacterial outer membrane and plays a role in pathogenesis by protecting micro-organisms from the lytic action of serum complement (Joiner, 1988). In S. typhi, LPS also interacts with intestinal epithelial cells during the initial stages of infection (Pier et al., 1998; Lyczak et al., 2001). 
LPS has a tripartite structure that includes lipid A, a core oligosaccharide and the $\mathrm{O}$-specific polysaccharide or $\mathrm{O}$ antigen (Schnaitman \& Klena, 1993; Raetz, 1996). The $\mathrm{O}$-antigen is the most surface-exposed LPS component and displays enormous structural variability, resulting in a large variety of serotypes (Reeves, 1993). S. typhi also produces a group I exopolysaccharide known as the $\mathrm{Vi}$ antigen, which is made of a homopolymer of high molecular mass (Virlogeux et al., 1996) and forms a capsular structure. The Vi antigen is found in virtually all clinical isolates from patients with acute typhoid infection. It protects $S$. typhi against complementmediated lysis as well as phagocytosis (Kossack et al., 1981).

The biosynthesis of exopolysaccharides is modulated by environmental factors through several regulatory systems (Virlogeux et al., 1996; Arricau et al., 1998; Whitfield \& Roberts, 1999). One of these regulatory components is the RcsC/RcsB two-component system, which consists of a cytoplasmic sensor kinase (RcsC) and cytosolic response regulator $(\mathrm{RcsB})$. More recently, a phosphorelay system involving the phosphotransmitter YojN was shown to be essential for transducing the signal from RcsC to RcsB in Escherichia coli (Takeda et al., 2001). It is quite possible that the same mechanism operates in $S$. typhi, since a homologue of Yoj $\mathrm{N}$ is also present in this bacterium. In E. coli, stimuli like osmotic shock (Sledjeski \& Gottesman, 1996) or growth at a low temperature (Whitfield \& Roberts, 1999) result in the phosphorylation of RcsB, which associates with the proteolytically labile protein RcsA forming a heterodimer that acts as a positive transcriptional regulator (Stout \& Gottesman, 1990). The activation of RcsC/YojN/RcsB-responsive promoters in E. coli and in other Enterobacteriaceae involves the recognition by the RcsB/RcsA dimer of a relatively conserved DNA sequence known as the 'RcsAB box' (Wehland \& Bernhard, 2000). This box, consisting of 14 base pairs (TaAGaatatTCctA), has been found in the upstream region of the promoter sequences of the colanic acid biosynthesis cluster of E. coli K-12, the K2 antigen cluster of Klebsiella pneumoniae, and the $\mathrm{Vi}$ antigen cluster of S. typhi (Wehland \& Bernhard, 2000).

The synthesis of LPS in S. typhi involves a large number of genes, the majority of which are organized in various clusters located on separate regions of the bacterial chromosome. It is conceivable that LPS gene expression at all of these various sites must be coordinated to ensure that all necessary components are available at any given time. Yet the regulation of LPS synthesis is not well understood. In E. coli and S. enterica serovar Typhimurium, a covalent substitution of lipid A with 4amino-4-deoxy-L-arabinose is regulated by the pmrA gene (Gunn \& Miller, 1996), which encodes a transcription factor that is activated during growth under mildly acidic conditions, in a PhoP/PhoQ-dependent manner during $\mathrm{Mg}^{2+}$ limitation, or by exposure to $\mathrm{Fe}^{3+}$ ions (Guo et al., 1997; Gunn et al., 1998; Ernst et al., 2001). This LPS modification reduces the net negative charge of the molecule, thus contributing to bacterial resistance to cationic peptides and presumably enhancing intracellular survival within phagosomes. Another level of regulation involves the regulation of gene expression of the core biosynthetic cluster by the $\mathrm{RfaH}$ protein (Farewell et al., 1991; Pradel \& Schnaitman, 1991) and also by the heat-shock response (Karow et al., 1991). RfaH is a homologue of the NusG factor that regulates gene expression of the haemolysin operon (Bailey et al., 1992; Leeds \& Welch, 1996, 1997), polysaccharide capsule genes (Stevens et al., 1997), the F plasmid tra operon (Beutin \& Achtman, 1979), and a gene involved in iron acquisition (Nagy et al., 2001). $\mathrm{RfaH}$ regulation occurs during transcript elongation and depends on a $5^{\prime}$-proximal, transcribed nucleic acid sequence known as ops (for operon polarity suppressor; Nieto et al., 1996; Bailey et al., 1997) that induces transcriptional pausing in vitro (Artsimovitch \& Landick, 2000) and in vivo (Leeds \& Welch, 1997). It has been recently demonstrated that $\mathrm{RfaH}$ recognizes RNApolymerase transcribing $\mathrm{RfaH}$-regulated operons by interacting with the ops sequence in the exposed nontemplate DNA strand of ops-paused transcription complexes (Artsimovitch \& Landick, 2002).

5 '-proximally transcribed sequences containing ops elements exist in the O-polysaccharide gene clusters of many enteric bacteria (Hobbs \& Reeves, 1994), suggesting that $\mathrm{RfaH}$ also plays a role in the regulation of the transcription elongation of $\mathrm{O}$-antigen genes. In a previous study, Marolda \& Valvano (1998) conducted a detailed analysis of the promoter region of the O7specific genes in E. coli. Using single-copy-number fusions to a reporter gene, these authors did not observe any detectable regulation of the $\mathrm{O} 7$-specific promoter at the level of initiation of transcription, concluding that regulation only occurs at the level of mRNA elongation in an RfaH-dependent manner.

Therefore, modulation of the cellular levels of $\mathrm{RfaH}$ may contribute to coordinate expression of $\mathrm{O}$-antigen and core LPS biosynthetic enzymes. Not much is known, however, about the regulation of the $\mathrm{rfaH}$ gene itself. In a previous study, we isolated the $r f a H$ gene from the $S$. typhi strain Ty2 and confirmed that its function is essential for LPS expression (Rojas et al., 2001). More importantly, we demonstrated that $r f a H$ gene expression varies with the growth phase, with the highest expression during stationary phase (Rojas et al., 2001). In this study, we provide evidence showing that regulation of $r f a H$ depends, at least in part, on the activity of the $\mathrm{R}$ poN alternative sigma factor and that differential $r f a H$ expression influences a similar pattern of $\mathrm{O}$-antigen production during the bacterial growth cycle.

\section{METHODS}

Bacterial strains, plasmids, media and growth conditions. Table 1 summarizes the properties of the bacterial strains and plasmids used in this study. Bacteria were grown in LuriaBertani medium (LB: Bacto tryptone, $10 \mathrm{~g} \mathrm{l}^{-1}$; Bacto yeast extract, $5 \mathrm{~g} \mathrm{l}^{-1} ; \mathrm{NaCl}, 5 \mathrm{~g} \mathrm{l}^{-1}$ ) or in minimal $\mathrm{E}$ medium $\left(\mathrm{MgSO}_{4} .7 \mathrm{H}_{2} \mathrm{O}, 0 \cdot 2 \mathrm{~g} \mathrm{l}^{-1}\right.$; citric acid monohydrate, $2 \mathrm{~g} \mathrm{l}^{-1}$; 
Table 1. S. typhi strains and plasmids used in this study

\begin{tabular}{|c|c|c|}
\hline Strain or plasmid & Relevant properties* & Source or reference \\
\hline \multicolumn{3}{|l|}{ Salmonella Typhi } \\
\hline Ty2 & Salmonella enterica serovar Typhi (S. typhi), wild-type & $\begin{array}{l}\text { Institute of Public Health, } \\
\text { Chile (ISP) }\end{array}$ \\
\hline M8 & Ty2 rfaH, $\mathrm{Cam}^{\mathrm{R}}$ & Rojas et al. (2001) \\
\hline M161 & Ty2 rpoN, Cam ${ }^{\mathrm{R}}$ & This study \\
\hline M159 & Ty $2 r c s B, \operatorname{Kan}^{\mathrm{R}}$ & This study \\
\hline MEI120 & Ty2 $\Phi\left(\right.$ hisD-lacZ), $\operatorname{Kan}^{\mathrm{R}}$ & Lyczak et al. (2001) \\
\hline \multicolumn{3}{|l|}{ Plasmids } \\
\hline pGEM-T Easy & Cloning vector, $\mathrm{Amp}^{\mathrm{R}}$ & Promega \\
\hline pSM334 & $\begin{array}{l}\text { pGEM-T Easy containing a } 334 \mathrm{bp} \text { fragment of the } y i g C-r f a H \text { intergenic } \\
\text { region from } S \text {. typhi Ty } 2\end{array}$ & This study \\
\hline pKD46 & pINT-ts derivative containing $\operatorname{araC}-P_{\text {araB }}$ and $\gamma \beta$ exo DNA fragments & Datsenko \& Wanner (2000) \\
\hline pKD4 & pANTS $\gamma$ derivative containing an FRT-flanked $\operatorname{Kan}^{\mathrm{R}}$ gene & Datsenko \& Wanner (2000) \\
\hline pKD3 & pANTS $\gamma$ derivative containing an FRT-flanked $\mathrm{Cam}^{\mathrm{R}}$ gene & Datsenko \& Wanner (2000) \\
\hline pFZY1 & Promoterless cloning vector, $\mathrm{Amp}^{\mathrm{R}} \mathrm{F}^{\prime} l a c$ replicon, $\operatorname{lac} \mathrm{Z}^{+} \mathrm{Y}^{+} A^{+}$ & Koop et al. (1987) \\
\hline pCE334 & $\begin{array}{l}\text { pFZY1 containing a } 334 \mathrm{bp} \text { fragment of the } y i g C-r f a H \text { intergenic region } \\
\text { from } S \text {. typhi Ty } 2\end{array}$ & This study \\
\hline pKHT19 & $\begin{array}{l}\text { pBluescript/KSII derivative containing a } 723 \mathrm{bp} \text { fragment including the } r f a H \\
\text { gene from S. typhi Ty2 }\end{array}$ & Rojas et al. (2001) \\
\hline
\end{tabular}

*Cam, chloramphenicol; Kan, kanamycin; Amp, ampicillin.

Table 2. Primers used in this study

\begin{tabular}{|ll|}
\hline Primer & \\
\hline WrcsB1 & CGG CGA ATT TGA AGA TTC CAC AGC ATT GAT CAA CAA CCT GTG TAG GCT GGA GCT GCT TCG \\
WrcsB2 & TTA ATG CTG CGG TTG AGC TTC TTG GCG ATT TCG GTG ACC ATT CCG GGG ATC CGT GCA CC \\
WrpoN1 & CAA CAG GCC ATC CGT CTG TTG CAG TTG TCT ACG CTG GAA CTG TGT AGG CTG GAG CTG \\
& CTT CG \\
WrpoN2 & CAG TAA TTT CGA CGT TAT GTC CGG TGA TAT TGA GCT GCA TAT GAA TAT CCT CCT TAG \\
RfaH2 & GTG ATA TTT GAT GGC GTC CAT TGT A \\
RfaHint2 & AAG GCC TTC GTT TTC CGC GTA CCA TTT TT \\
WbaP51 & CTC CCC GGG AAT GGA TAA TAT TGA TAA TAA G \\
WbaP592 & TCG GAT ATC TTA ATA CGC ACC ATC TGC CC \\
HisG1 & CCG GAT CCA GAC AAC ACC CGC TTA CGC ATA G \\
HisG2 & CCG GAT CCT CTC CAT GGT TTC CCA GAA CAA C \\
\hline
\end{tabular}

$\mathrm{K}_{2} \mathrm{HPO}_{4} \cdot 3 \mathrm{H}_{2} \mathrm{O}, \quad 13 \cdot 1 \mathrm{~g} \mathrm{l}^{-1} ; \quad \mathrm{NaNH}_{4} \mathrm{HPO}_{4} \cdot 4 \mathrm{H}_{2} \mathrm{O}, \quad 3 \cdot 3 \mathrm{~g} \mathrm{l}^{-1}$ ) containing $0 \cdot 2 \%$ glucose as carbon source. Low-nitrogen $\mathrm{E}$ medium contained $1 \%$ of the amount of nitrogen in E medium. Minimal media were supplemented with $50 \mu \mathrm{g}$ cysteine $\mathrm{ml}^{-1}$ and $50 \mu \mathrm{g}$ tryptophan $\mathrm{ml}^{-1}$. Media were supplemented with $100 \mu \mathrm{g}$ ampicillin $\mathrm{ml}^{-1}, 20 \mu \mathrm{g}$ chloramphenicol $\mathrm{ml}^{-1}$ or $50 \mu \mathrm{g}$ kanamycin $\mathrm{ml}^{-1}$ as appropriate.

Construction of a lac $Z$ transcriptional fusion to the $\mathrm{rfaH}$ gene. The S. typhi Ty 2 raH promoter region was amplified by PCR using the rfaH2 and rfaHint 2 primers (Table 2), which were designed according to the DNA sequence information available for the S. typhi strain CT18 (Parkhill et al., 2001). A $334 \mathrm{bp}$ amplicon was cloned into the vector pGEM-T Easy, to yield plasmid pSM334. The 334 bp insert in pSM334 was then transferred into the plasmid pFZY1. This is a single-copy- number vector designed for the construction of transcriptional fusions to the lac operon (Koop et al., 1987). The resulting plasmid, pCE334, was transformed into S. typhi Ty2. The pFZY1 vector was also transformed as a negative control.

Mutagenesis of S. typhi rcsB and rpoN genes. Mutagenesis was performed according to the method described by Datsenko \& Wanner (2000) to disrupt specific chromosomal genes using PCR products. For this purpose, S. typhi Ty 2 was transformed with pKD46, a temperature-sensitive, low-copynumber plasmid that expresses the phage $\lambda$ Red recombinase system under the control of the arabinose-inducible $\mathrm{P}_{\text {araB }}$ promoter. The Red-mediated recombination is required to replace the targeted chromosomal sequence with an antibioticresistance gene that is generated by PCR. S. typhi Ty 2 cells 
carrying pKD46 were transformed by electroporation with a PCR product that was generated using either plasmid $\mathrm{pKD} 3$ or pKD4 as templates. The plasmid pKD3 carries a chloramphenicol acetyltransferase gene flanked by FRT sites while pKD4 carries a gene encoding kanamycin resistance, also flanked by FRT sites. Primers WrpoN1 and WrpoN2 were used with the pKD3 template to obtain a product for disruption of $r p o N$. Primers WrcsB1 and WrcsB2 were used with the pKD4 template to obtain a product for disruption of $r c s B$. The sequences of the primers are shown in Table 2. Transformants were plated on LB agar plates containing chloramphenicol, for the selection of rpoN mutants, or kanamycin, for the selection of $r c s B$ mutants. Transformants were replica plated in the absence of antibiotic selection at $43^{\circ} \mathrm{C}$ and then assayed for ampicillin sensitivity, to confirm the loss of pKD46.

RT-PCR. For expression analysis, each strain was grown to the selected $\mathrm{OD}_{600}$ in $50 \mathrm{ml}$ LB. RNA was extracted using the standard TRIzol procedure. After DNase I treatment, RNA was reverse transcribed using SuperScript II $\left(200 \mathrm{U} \mathrm{\mu l}^{-1}\right)$ and antisense primers for his $G$ and wbaP (HisG2 and WbaP592, respectively). Single-stranded DNA was then amplified using the primers for genes: his G (HisG1 and HisG2) and wbaP (WbaP591 and WbaP592). The sequences of the primers are indicated in Table 2 . The PCR products were analysed by electrophoresis on $1.5 \%$ agarose gels.

LPS analysis. Culture samples were adjusted to $\mathrm{OD}_{600} 2 \cdot 0$ in a final volume of $100 \mu \mathrm{l}$. Then, proteinase-K-digested whole-cell lysates were prepared as described by Hitchcock \& Brown (1983) and LPS was separated on $14 \%$ acrylamide gels using a Tricine/SDS buffer system (Lesse et al., 1990). Gel loadings were normalized so that each sample represented the same number of cells. Each well was loaded with approximately $1 \times 10^{8}$ c.f.u. Gels were silver stained by a modification of the procedure of Tsai \& Frasch (1982). Densitometric analyses of the gels were performed using the UN-SCAN-IT gel software (Silk Scientific).

$\boldsymbol{\beta}$-Galactosidase assays. Bacteria were grown overnight in LB or minimal E medium, subcultured and grown in $100 \mathrm{ml}$ of the same medium on an orbital shaker. Every $30 \mathrm{~min}$, a $2 \mathrm{ml}$ sample was withdrawn to measure the bacterial growth $\left(\mathrm{OD}_{600}\right.$ and c.f.u. $\left.\mathrm{ml}^{-1}\right)$ and the $\beta$-galactosidase activity according to Miller (1972). Enzyme activities (Miller units), normalized for cell density $\left(\mathrm{OD}_{600}\right)$, were calculated using the equation $\left[\left(A_{420}-1.75 A_{550}\right) \times 1000\right] /[$ reaction time (min) $\times$ culture volume $\left.(\mathrm{ml}) \times \mathrm{OD}_{600}\right]$. Each sample was analysed in triplicate during at least three independent experiments.

\section{RESULTS}

\section{Expression of S. typhi LPS during growth correlates with $r f a H$ transcription}

It is well documented that the $\mathrm{RfaH}$ elongation factor positively regulates the LPS core and O-polysaccharide genes. The environmental signals, however, that modulate the expression of the $r f a H$ gene are largely unknown. In a previous study, we used RT-PCR analysis to test the effects of various growth conditions on the transcription of $r f a H$ (Rojas et al., 2001). We demonstrated that the transcription of $r f a H$ in S. typhi Ty2 displays a growthphase-dependent regulation, with maximal expression during late-exponential and stationary phases. In contrast, we found that environmental factors that are known to be important for LPS regulation in other systems, such as growth temperature (Al-Hendy et al., 1991) and osmolarity (Aguilar et al., 1997), do not affect transcription of the $\mathrm{rfaH}$ gene in cells grown to late exponential phase (Rojas et al., 2001). To determine whether the transcriptional regulation of the $\mathrm{rfaH}$ gene is exerted at the promoter level we constructed a transcriptional fusion of the $\mathrm{rfaH}$ promoter with a promoterless lac operon in a single-copy-number plasmid (pFZY1). The resulting plasmid, pCE334, was transformed into $S$. typhi Ty2. The pFZY1 vector was also transformed as a negative control. The production of $\beta$-galactosidase in strain Ty $2 /$ pCE334 was assayed during the various stages of growth in rich medium at $37^{\circ} \mathrm{C}$. The units of enzyme activity were corrected for the background levels determined in strain Ty2/pFZY1, which ranged between 0.5 and 5.0 Miller units during growth in LB medium. Fig. $1(\mathrm{a})$ shows that $\beta$-galactosidase production, driven by the $\mathrm{rfaH}$ promoter region, increases at the late exponential growth phase, reaching maximal expression during stationary phase. These results are consistent with the idea that growthregulated expression of $r f a H$ is exerted at the level of transcription. To rule out the possibility that the upregulation of the $r f a H$ gene was a general growthphase-dependent phenomenon, we assayed the production of $\beta$-galactosidase in S. typhi strain MEI120, which contains a $M u d J(k a n, l a c)$ insertion in the hisD gene (Lyczak et al., 2001). Fig. 1(a) shows that the his D-lac Z expression does not change in response to the bacterial growth phase.

To examine whether the growth-regulated expression of $r f a H$ has any biological significance in terms of LPS synthesis, we monitored the formation of S. typhi LPS during growth. Fig. 1(b) shows that the production of O-specific LPS varies during the bacterial growth. During the exponential phase (lanes 1 and 2), a very small amount of $\mathrm{O}$-antigen is observed. As cells reach the late exponential phase, the formation of $\mathrm{O}$-antigen increases (lanes 3, 4 and 5), and it is maximally expressed at the stationary phase (lanes 6 and 7). Densitometric analysis of the LPS gel in Fig. 1(b) revealed that the ratio of $\mathrm{O}$-antigen to the lipid A-core region in the sample grown to $\mathrm{OD}_{600} 1.239$ (Fig. 1b, lane 7) was approximately twofold $(183 \%)$ higher than that of the sample grown to $\mathrm{OD}_{600} 0 \cdot 157$ (Fig. 1b, lane 1). The pattern of O-antigen expression parallels the growthregulated expression of $r f a H$, suggesting that the formation of O-antigen LPS by the bacterial cells during growth is modulated by the cellular levels of $\mathrm{RfaH}$. In support of this notion, no changes in O-antigen expression during growth were observed when S. typhi Ty2 was transformed with the high-copy-number plasmid pKHT19, harbouring the $r f a H$ gene (data not shown). Similar results were obtained when an $r f a H$ null mutant was transformed with pKHT19.

The first step in the synthesis of the $\mathrm{O}$-antigen in species of Salmonella involves the addition of galactose 1phosphate onto undecaprenol-P to produce a galactose$\mathrm{P}$-P-undecaprenol intermediate that is strictly required 


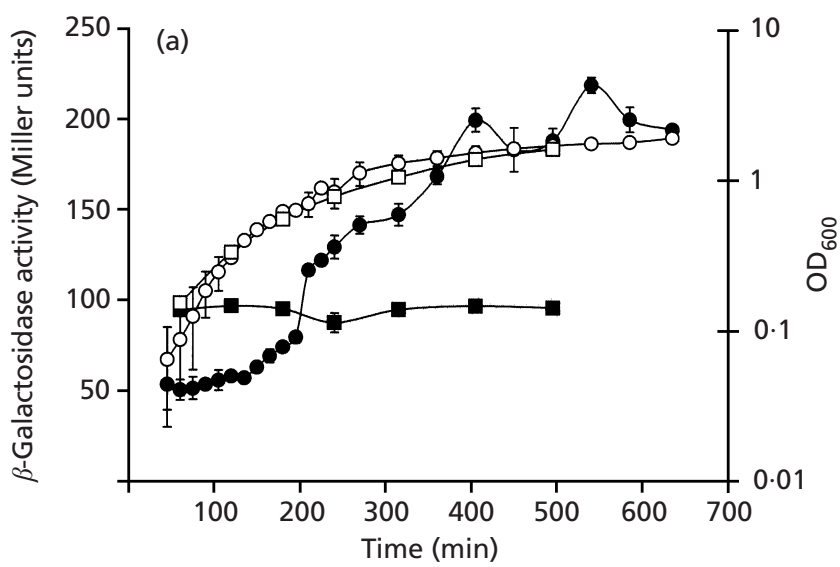

(b)

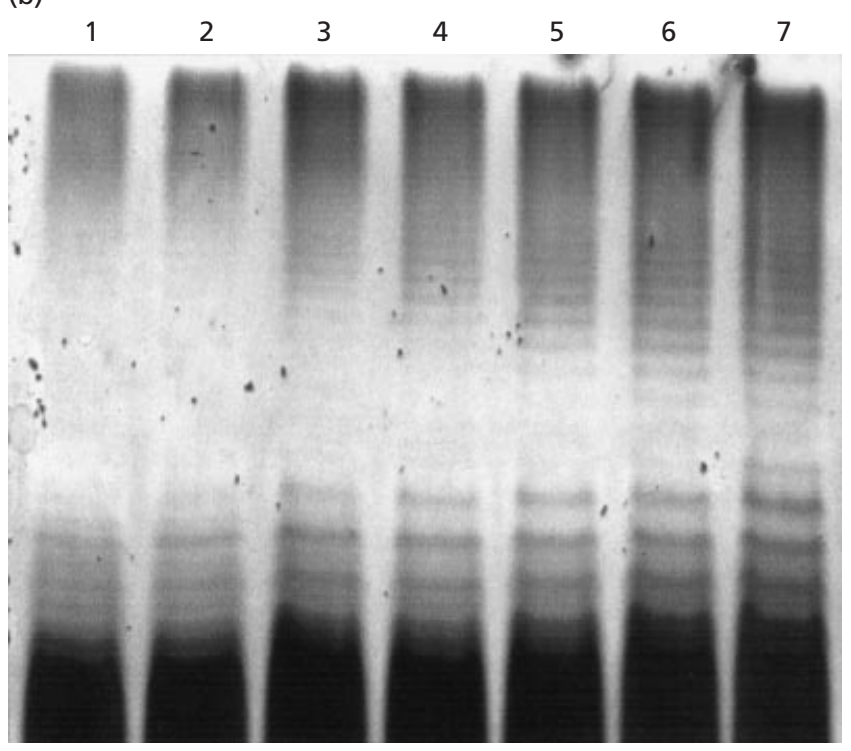

Fig. 1. Expression of $r f a H$ and production of LPS in S. typhi Ty2/pCE334 at different points of the growth curve in LB at $37^{\circ} \mathrm{C}$. (a) Growth curve of S. typhi Ty2/pCE334 and expression of $r f a H-l a c Z$ transcriptional fusion. $O$, Growth curve $\left(\mathrm{OD}_{600}\right)$; $\beta$-galactosidase activity of the $\mathrm{rfaH}-\mathrm{lacZ}$ fusion; $\square$, growth of strain MEI120 (hisD-lacZ fusion) used as control; $\square, \beta$ galactosidase activity of the hisD-lacz fusion. Data are the mean \pm SD of three independent assays. (b) LPS profiles of cells grown to the following $\mathrm{OD}_{600}$ : lane 1, 0.157; lane 2, 0.353; lane 3, 0.462; lane 4, 0.572; lane 5, 0.775; lane 6, 1.004; lane 7, 1.239. All samples were adjusted to an $\mathrm{OD}_{600}$ of 2.0 in a final volume of $100 \mu \mathrm{l}$ for LPS preparation. LPS was analysed by Tricine/SDSPAGE on a $14 \%$ acrylamide gel. Each well was loaded with approximately $1 \times 10^{8}$ c.f.u.

for the assembly of the O-antigen unit (Wang \& Reeves, 1994; Wang et al., 1996). This reaction is catalysed by the product of the wbaP gene, which is the terminal gene in the O-antigen cluster. Thus, the level of transcription of wbaP can be used to monitor whether $\mathrm{RfaH}$ modulates the expression of the entire S. typhi Oantigen gene cluster directly. We examined by RT-PCR the levels of wbaP mRNA in cells grown to midexponential phase and stationary phase. As a control, we also determined the mRNA levels of hisG. Fig. 2

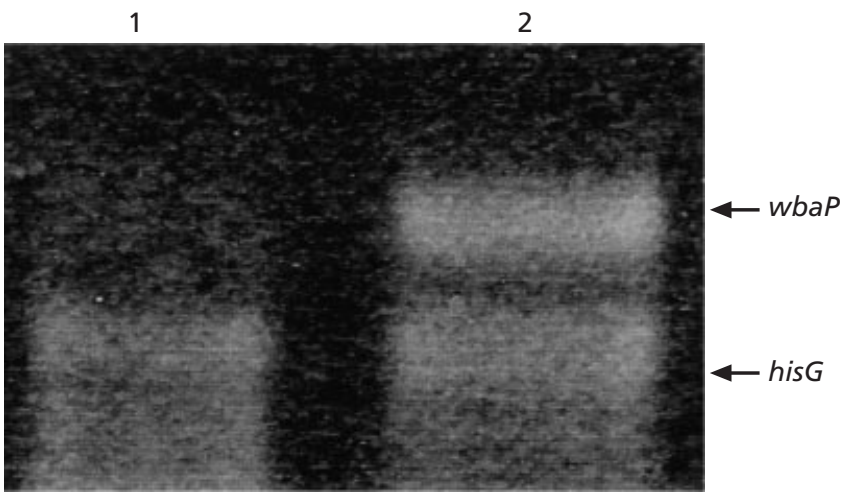

Fig. 2. Expression of wbaP mRNA in S. typhi Ty2 at the exponential and stationary phases of growth. mRNA levels of wbaP and his were examined by RT-PCR in samples obtained at mid-exponential phase $\left(\mathrm{OD}_{600} \mathbf{0} \cdot \mathbf{2}\right.$, lane 1) and stationary phase $\left(O D_{600} 1 \cdot 0\right.$, lane 2$)$. Arrows indicate $R T-P C R$ products using wbaP-and hisG-specific primers. The PCR products were analysed by electrophoresis on a $1.5 \%$ agarose gel.

shows the mRNA levels of wbaP and his $G$ genes at different stages of growth as assessed by RT-PCR, in the wild-type Ty 2 strain. The densitometric quantification of the lanes demonstrated that the expression of $w b a P$ relative to the expression of hisG is low in midexponential phase but increases significantly (over 6.0fold) when cells reach stationary phase (data not shown). The expression of his $G$ does not change in response to the bacterial growth phase (Fig. 1a and data not shown). The wbaP-specific mRNA levels in strain Ty2/pKHT19, which overexpresses the $\mathrm{RfaH}$ protein, remained unchanged during growth (data not shown). No wbaP transcription was detected in the $\mathrm{rfaH}$ null mutant. Taken together, our results indicate that the growthphase regulation of $\mathrm{O}$-antigen expression is associated with the growth-phase expression of $\mathrm{RfaH}$. In contrast, the production of the lipid A-core region was not affected similarly, since a complete lipid A-core band was observed at all different stages of growth (Fig. 1b). This is not completely unexpected, since in a previous study, Marolda \& Valvano (1998) have shown that $\mathrm{RfaH}$-mediated regulation of the core operons in E. coli and Salmonella is less tight than the regulation exerted in the $\mathrm{O}$-antigen operon. These differences in $\mathrm{RfaH}-$ mediated regulation may be due to structural differences that exist in the regions surrounding the ops elements in the $\mathrm{O}$-antigen and core promoter regions (Marolda \& Valvano, 1998).

\section{Characterization of the $\mathrm{rfaH}-\mathrm{yigC}$ promoter region}

To investigate the factors influencing expression of the $\mathrm{rfaH}$ gene, the DNA sequence of the $334 \mathrm{bp}$ insert in pCE334 was determined. The sequence was identical to that reported for the same region in S. typhi CT18 (www.sanger.ac.uk/Projects/S.typhi/blast_server. shtml). This fragment includes $128 \mathrm{bp}$ from the $\mathrm{rfaH}$ 


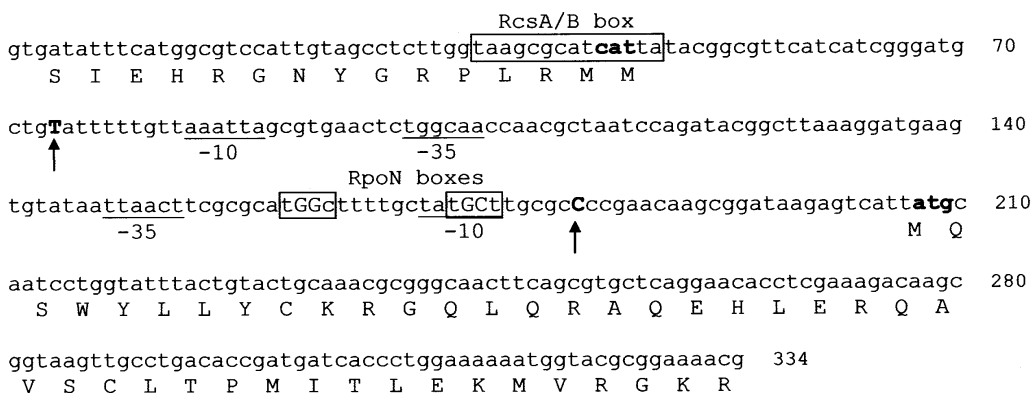

Fig. 3. Sequence analysis of the yigC-rfaH intergenic promoter region using the Natural Network Promoter Prediction program. Arrows indicate the predicted transcription initiation sites for $\mathrm{rfaH}\left(\mathrm{C}_{182}\right)$ and for yigC $\left(\mathrm{T}_{74}\right)$. Underlined sequences indicate the predicted -35 and -10 promoter regions for yigC (bases 84-105) and for $\mathrm{rfaH}$ (bases 148-176). Boxed segments indicate the putative RcsAB and RpoN boxes. Predicted translational start sites for $y i g \mathrm{C}$ and $\mathrm{rfaH}$ are as labelled.

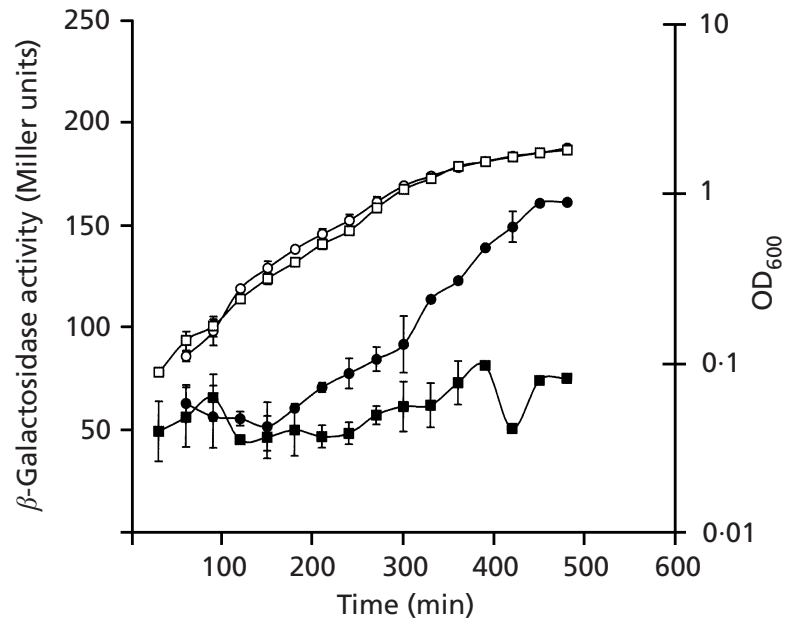

Fig. 4. Growth and expression of $r f a H-l a c Z$ transcriptional fusion in S. typhi rpoN/pCE334 and rcsB/pCE334 mutants. $\bigcirc, \square$, Growth $\left(\mathrm{OD}_{600}\right)$ of $S$. typhi $\mathrm{rcsB} / \mathrm{pCE} 334(\mathrm{O})$ and $S$. typhi $r$ roN/pCE334 $(\square)$; $\mathbf{0}, \mathbf{\square}$, expression of $r f a H-l a c Z$ fusions in $S$. typhi rcsB (O) and S. typhi rpoN (ם) mutants, measured as $\beta$-galactosidase activity. Data are the mean \pm SD of three independent assays.

coding region and also $46 \mathrm{bp}$ from the coding region of the upstream gene, $y$ igC, which is transcribed in the opposite direction (Fig. 3). Therefore, the $334 \mathrm{bp}$ fragment spans two divergently transcribed promoters.

The sequence of the intergenetic region was analysed with the Natural Network Promoter Prediction program (www.fruitfly.org/seq_tools/promoter.html) to predict potential promoter sites. The strongest predictions corresponded to positions 84 and 105 for the yigC promoter (score 0.84 ), and to positions 148 and 176 for the $r f a H$ promoter (score $0 \cdot 88$ ). The program also predicted the location of the initiation of transcription at bases $\mathrm{C}_{182}$ and $\mathrm{T}_{74}$ for the $r f a H$ and yigC transcripts, respectively (Fig. 3).

A closer examination of the sequences upstream of the $r f a H$ promoter revealed homologies to potential binding sites of known regulatory proteins. The sequence $5^{\prime}-$ TAAGCGCATCATTA-3' (Fig. 3) had similarities with an RcsAB binding box, described in the upstream regions of promoter elements regulated by the RcsB/ RcsA dimer (Wehland \& Bernhard, 2000). Furthermore, sequences containing the conserved motifs $5^{\prime}$-TGCT- $3^{\prime}$ and $5^{\prime}$-TGGC-3' were also found (Fig. 3). These sequences resemble the -12 and -24 recognition regions for the alternative sigma factor $\mathrm{RpoN}$, which is responsible for gene expression under nitrogen starvation (Shingler, 1996; Wang \& Gralla, 1998).

\section{RpoN regulates $\mathrm{rfaH}$ gene expression}

To investigate whether RpoN or the RcsC/YojN/RcsB system could regulate the expression of $r f a H$, we constructed insertional mutations in the $r p o N$ and $r c s B$ genes of S. typhi Ty2 as described in Methods. The two mutant strains, named M161 (Ty2 rpoN) and M159 (Ty2 rcsB), were transformed with plasmid pCE334 and the $\beta$-galactosidase activity was measured during the bacterial growth. Fig. 4 shows that inactivation of $r p o N$ abolished the growth-phase-dependent pattern of $\beta$ galactosidase production. In contrast, the $r c s B$ mutant strain displayed a pattern of $\beta$-galactosidase production over the course of the growth experiment that was similar to that of the wild-type S. typhi Ty2 (Fig. 1a). These results suggest that the $r f a H$ promoter activity can be modulated in an RpoN-dependent manner. To support this notion, the production of LPS by the mutant strain was also examined. Fig. 5(a) shows that the LPS profiles of the rcsB mutant remain identical to those of the wild-type strain (Fig. 1b). Densitometric analyses also revealed an approximately twofold increased proportion of $\mathrm{O}$-antigen relative to lipid A-core in the samples obtained from cells grown to stationary phase (data not shown). In contrast, the proportion of $\mathrm{O}$-antigen relative to lipid A-core was constant in the LPS profiles of the rpoN mutant irrespective of the growth stage (Fig. 5b).

\section{Nitrogen limitation increases the transcription of the $r f a H$ gene and the production of O-specific LPS}

The results of the previous section demonstrate that expression of $r f a H$ in S. typhi Ty2 is regulated, either directly or indirectly, by the alternative sigma factor RpoN. Several cellular functions are regulated by RpoN, 
(a)

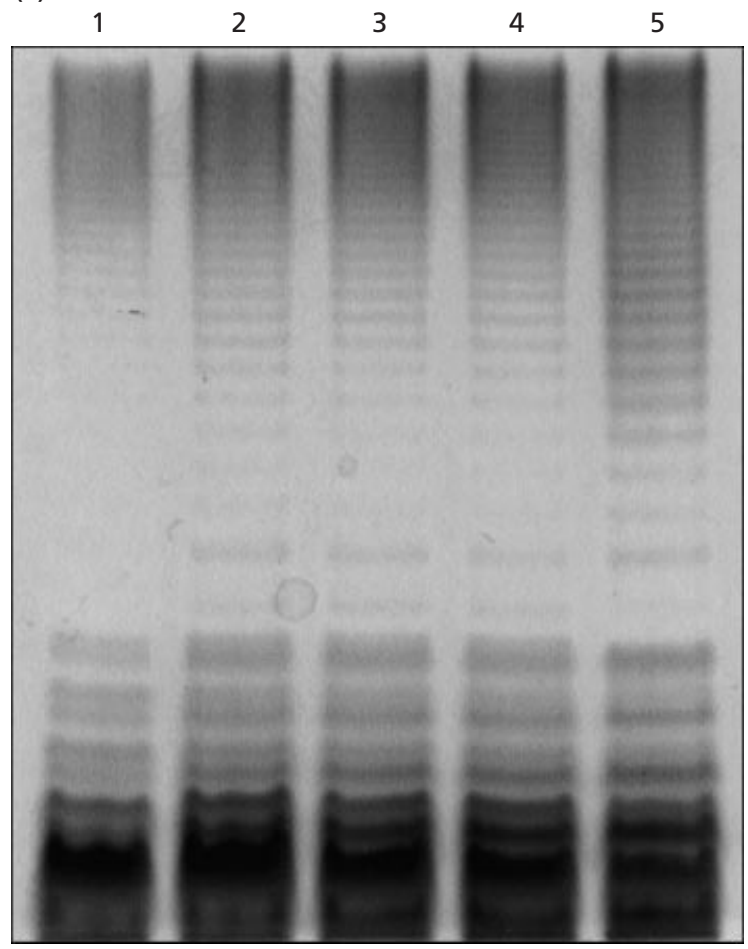

(b)

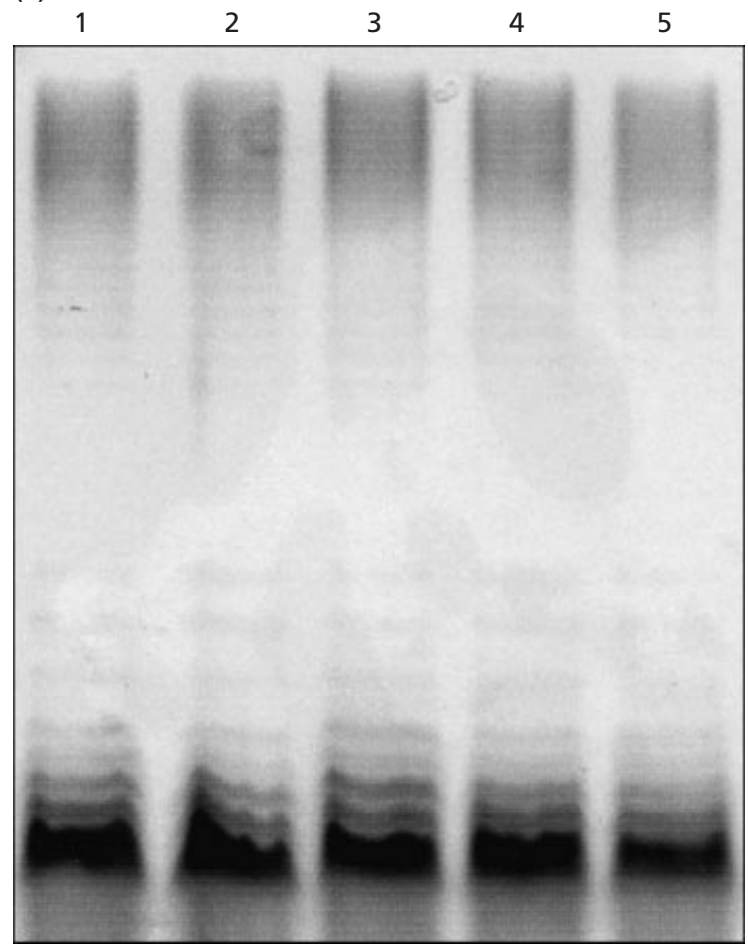

Fig. 5. Production of LPS in S. typhi $r c s B / p C E 334$ and S. typhi rpoN/pCE334 mutants grown to different points of the growth curve in LB. (a) LPS profiles of S. typhi rcsB/pCE334 grown to the following $\mathrm{OD}_{600}$ : lane 1, 0.100; lane 2, 0.186; lane 3, 0.445; lane 4, 0.668; lane 5, 1.021. (b) LPS profiles of S. typhi rpoN/pCE334 grown to the following $\mathrm{OD}_{600}$ : lane 1, 0.104; lane 2, 0.256; lane $3,0.401$; lane 4, 0.676; lane 5, 1.181. All samples were adjusted to an $\mathrm{OD}_{600}$ of 2.0 in a final volume of $100 \mu \mathrm{l}$ for LPS including utilization of various nitrogen and carbon sources, energy metabolism, chemotaxis, flagellation, and response to heat shock (reviewed by Buck et al., 2000). But to our knowledge, a role for RpoN in regulation of LPS biosynthesis has not been described. Because of our results demonstrating that the growthdependent regulation of the $r f a H$ gene requires the RpoN function, we reasoned that $r f a H$ expression would also be regulated by nitrogen limitation. To test this hypothesis, we grew $S$. typhi/pCE334 in lownitrogen minimal $\mathrm{E}$ medium and measured the $\beta$ galactosidase activity in the exponential phase of growth $\left(\mathrm{OD}_{600} 0 \cdot 2\right)$. As shown in Table 3, expression of the $\mathrm{rfaH}-\mathrm{lacZ}$ fusion increased nearly fourfold when bacteria were grown in the low-nitrogen medium. Additionally, we investigated the effect of nitrogen limitation on LPS production by the wild-type Ty2 strain grown to mid-exponential phase $\left(\mathrm{OD}_{600} 0 \cdot 2\right)$. Fig. 6 (lane 2) shows that nitrogen limitation increased the expression of the O-antigen by the wild-type S. typhi Ty2, as demonstrated by a similar profile to that observed during the stationary phase of growth in rich medium (Fig. 1b). The relationship between LPS production and nitrogen limitation parallels the pattern of $r f a H$ regulation under the control of $\mathrm{RpoN}$ and is consistent with the idea that RpoN modulates LPS formation via its effect on $r f a H$ gene expression during bacterial growth.

\section{DISCUSSION}

Bacterial cells can sense host microenvironments and respond by coordinately regulating gene expression. Invasion of the intestinal epithelium and survival within macrophages are essential properties required for typhoidal pathogenesis. Therefore, Salmonella must sense the host environment, either at mucosal surfaces or within host cells, and respond by inducing or repressing gene expression. Essential to the adaptation of bacteria during host infection is the remodelling of the bacterial surface (Ernst et al., 2001). This involves the modification of outer-membrane proteins and of LPS, the most abundant component of the bacterial envelope and a major pathogenic factor of Salmonella (Joiner, 1988; Rosenberger et al., 2000; Ernst et al., 2001). LPS is required for complement evasion (Joiner, 1988) as well as for invasion of intestinal epithelial cells (Pier et al., 1998; Lyczak et al., 2001) in the pathogenesis of S. typhi.

Evidence from other studies has demonstrated that the transcription of the LPS core and O-polysaccharide gene clusters is subject to positive regulation at the level of mRNA elongation by the RfaH protein (Farewell et al., 1991; Pradel \& Schnaitman, 1991; Bailey et al., 1996; Marolda \& Valvano, 1998; Wang et al., 1998). $\mathrm{RfaH}$ promotes expression of operons encoding proteins

preparation. LPS was analysed by Tricine/SDS-PAGE on $14 \%$ acrylamide gels. Each well was loaded with approximately $1 \times 10^{8}$ c.f.u. 
Table 3. Effect of nitrogen availability on the expression of $r f a H-l a c Z$ transcriptional fusion

Bacteria were grown in the indicated medium to $\operatorname{OD}_{600} 0 \cdot 2$. Values are the mean \pm SD of three independent assays. ND, Not determined because the rpoN mutant strain (M161/pCE334) does not grow in low-nitrogen E medium.

\begin{tabular}{|lccc|}
\hline Strain & \multicolumn{3}{c|}{$\boldsymbol{\beta}$-Galactosidase activity (Miller units) } \\
\cline { 2 - 4 } & LB & E medium & $\begin{array}{c}\text { Low-nitrogen } \\
\text { E medium }\end{array}$ \\
\hline Ty2/pCE334 & $55 \cdot 34 \pm 4 \cdot 58$ & $65 \cdot 02 \pm 4 \cdot 78$ & $201 \cdot 64 \pm 45 \cdot 17 *$ \\
M161/pCE334 & $45 \cdot 11 \pm 7 \cdot 89$ & $60 \cdot 23 \pm 9 \cdot 28$ & ND \\
\hline
\end{tabular}

*Significantly different from Ty2/pCE334 grown in LB $(P<0 \cdot 01$, ANOvA and Dunnet).

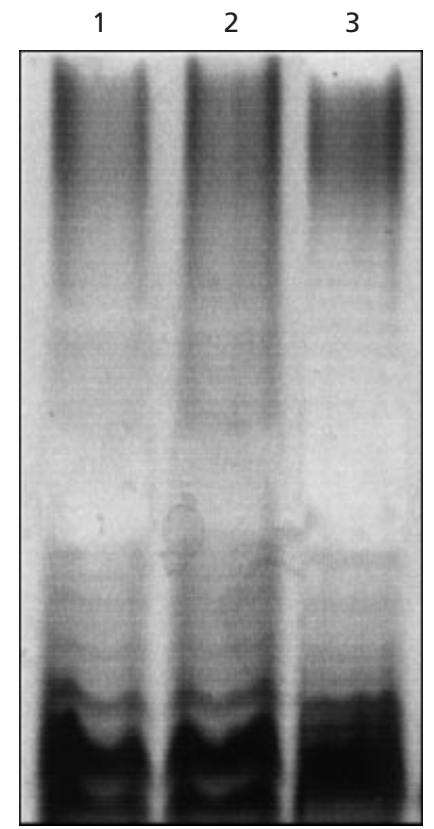

Fig. 6. Effect of nitrogen availability on LPS production by $S$. typhi Ty2 and S. typhi rpoN, analysed by Tricine/SDS-PAGE. Cultures were grown to mid-exponential phase. Samples were adjusted to an $\mathrm{OD}_{600}$ of 2.0 in a final volume of $100 \mu \mathrm{l}$ for LPS preparation. LPS was analysed by Tricine/SDS-PAGE on $14 \%$ acrylamide gels. Each well was loaded with approximately $1 \times 10^{8}$ c.f.u. Lane 1, S. typhi Ty2 grown in E medium; lane 2, S. typhi Ty2 grown in low-nitrogen $\mathrm{E}$ medium; lane 3, S. typhi rpoN grown in E medium.

targeted to the cell surface or membrane (Bailey et al., 1997) by promoting the efficient elongation of the mRNA (Artsimovitch \& Landick, 2002). Based on a detailed deletion-fusion analysis of the E. coli O7 LPS promoter region, Marolda \& Valvano (1998) have proposed a model involving premature termination of transcription relieved by the $\mathrm{RfaH}$ protein that operates to regulate the expression of $\mathrm{O}$-specific polysaccharide genes. In this model, the site for premature transcription termination is located within a relatively long untranslated $5^{\prime}$ mRNA, and possibly depends on the formation of a hairpin. A similar $5^{\prime}$ untranslated region is present in the case of the Salmonella O-antigen clusters. Therefore, it is reasonable to assume that a similar RfaH-dependent regulation of transcription elongation takes place in the S. typhi O-antigen gene cluster.

In this study, we have shown that the synthesis of the $S$. typhi $\mathrm{O}$-antigen is regulated by $\mathrm{RfaH}$ in a growth-phasedependent manner. Production of the $\mathrm{O}$-antigen correlates with the differential expression of the $r f a H$ gene during the bacterial growth, increasing at the late exponential phase and reaching maximal expression at the stationary phase. As has been shown in other bacteria (Marolda \& Valvano, 1998; Wang et al., 1998), we found that $\mathrm{RfaH}$ modulates the $\mathrm{O}$-antigen genes by positively regulating gene transcription, as demonstrated by the increase in the $w b a P$-specific mRNA levels at the stationary phase of growth.

Previous work in our laboratory has shown that environmental conditions that are known to be important for LPS regulation in other systems, such as growth temperature (Al-Hendy et al., 1991) and osmolarity (Aguilar et al., 1997), do not affect transcription of the $r f a H$ gene (Rojas et al., 2001). In this study, we examined the sequences upstream of the $r f a H$ promoter and found potential binding sites for the RcsB/RcsA dimer of the RcsC/YojN/RcsB phosphorelay system and for the $\mathrm{RpoN}$ alternative sigma factor. We therefore investigated the transcriptional activity of the $r f a H$ gene under growth conditions that activate the RcsC/YojN/ $\mathrm{RcsB}$ system or $r p o N$-mediated transcription. No effects were detected with osmotic shock or growth at low temperature, which are known to induce the expression of exopolysaccharides by activating the $\mathrm{RcsC} / \mathrm{RcsB}$ system (Sledjeski \& Gottesman, 1996; Whitfield \& Roberts, 1999). Nor were changes in $\mathrm{rfaH}$ expression resulting from a mutation in the $r c s B$ gene found. In contrast, growth of $S$. typhi Ty2 in a nitrogen-limited medium induced $\mathrm{rfaH}$ gene transcription, and the 
inactivation of the rpoN gene, which encodes the alternative sigma factor $\sigma^{54}\left(\sigma^{\mathrm{N}}\right)$, abolished the growthphase-dependent induction of $r f a H$ expression.

Most bacteria possess one copy of $r p o N$, which generally is constitutively expressed and not essential for survival and growth under favourable conditions (Buck et al., 2000). RpoN is a specialized sigma factor that recognizes a subset of promoters in bacteria that control regulation of nitrogen metabolism as well as many other biological activities, transcribing genes with diverse physiological roles, including flagellation, chemotaxis, energy metabolism, RNA modification, electron transport, response to heat shock and expression of alternative sigma factors (Merrick, 1993; Buck et al., 2000). RpoN-mediated transcription has also been associated with bacterial pathogenicity. Early reports showed that expression of pilin genes in Pseudomonas aeruginosa (Ishimoto \& Lory, 1989) and in Neisseria gonorrhoeae (Klimpel et al., 1989) required RpoN. More recently, Klose \& Mekalanos (1998) reported that an rpoN null mutant of Vibrio cholerae was defective for colonization in an infant mouse model of cholera, and that this defect was distinct from the non-motile and glutamine synthetase phenotypes of the rpoN mutant (Klose \& Mekalanos, 1998). Other authors have shown that a strain of Proteus mirabilis carrying a mutation in a gene which is highly homologous to ORF284 of the rpoN operon has a reduced ability to infect the urinary tract of CBA mice (Zhao et al., 1999).

A role for RpoN in the expression of cell-surface polysaccharides has been demonstrated in the case of alginate production by Pseudomonas aeruginosa (Boucher et al., 2000), but to our knowledge this is the first observation that RpoN plays a role in modulation of gene expression of O-antigen LPS genes. Our results suggest that RpoN acts directly or indirectly on $r f a H$ gene expression to modulate $\mathrm{O}$-antigen synthesis in an $\mathrm{RfaH}$-mediated fashion. This regulation is manifested not only during the growth cycle but also under conditions of nitrogen limitation. Further studies are under way to characterize in detail the mechanism of $\mathrm{RpoN}$ action on the $r f a H$ promoter region and the possible interrelation between stationary phase and nitrogen limitation in relation to $\mathrm{O}$-antigen synthesis.

\section{ACKNOWLEDGEMENTS}

This investigation was supported by grants 1990967 and 7990058 from FONDECYT to I.C. and grant MOP10206 from the Canadian Institutes of Health Research to M.A.V.

\section{REFERENCES}

Aguilar, A., Merino, S., Rubires, X. \& Tomas, J. M. (1997). Influence of osmolarity on lipopolysaccharide and virulence of Aeromonas hydrophila serotype O:34 strains grown at $37^{\circ} \mathrm{C}$. Infect Immun 65, 1245-1250.

Al-Hendy, A., Toivanen, P. \& Skurnik, M. (1991). The effect of growth temperature on the biosynthesis of Yersinia enterocolitica
$\mathrm{O}: 3$ lipopolysaccharide: temperature regulates the transcription of the $r f b$ but not the $r f a$ region. Microb Pathog 10, 81-86.

Arricau, N., Hermant, D., Waxin, H., Echobicon, C., Duffey, P. S. \& Popoff, M. Y. (1998). The RcsB and RcsC regulatory system of Salmonella typhi differentially regulates the expression of invasion proteins, flagellin and $\mathrm{Vi}$ antigen in response to osmolarity. Mol Microbiol 29, 835-850.

Artsimovitch, I. \& Landick, R. (2000). Pausing by bacterial polymerase is mediated by mechanistically distinct classes of signals. Proc Natl Acad Sci U S A 97, 7090-7095.

Artsimovitch, I. \& Landick, R. (2002). The transcriptional regulator $\mathrm{RfaH}$ stimulates RNA chain elongation after recruitment to elongation complexes by the exposed nontemplate DNA strand. Cell 109, 193-203.

Bailey, M. J. A., Koronakis, V., Schmoll, T. \& Hughes, C. (1992). Escherichia coli HlyT protein, a transcriptional activator of haemolysin synthesis and secretion, is encoded by the $r f a H(s f r B)$ locus required for expression of sex factor and lipopolysaccharide genes. Mol Microbiol 6, 1003-1012.

Bailey, M. J. A., Hughes, C. \& Koronakis, V. (1996). Increased distal gene transcription by the elongation factor $r f a H$, a specialized homologue of NusG. Mol Microbiol 22, 729-737.

Bailey, M. J. A., Hughes, C. \& Koronakis, V. (1997). RfaH and the ops element, components of a novel system controlling bacterial transcription elongation. Mol Microbiol 26, 845-851.

Beutin, L. \& Achtman, M. (1979). Two Escherichia coli chromosomal cistrons, $s f r A$ and $s f r B$, which are needed for expression of F factor tra functions. J Bacteriol 139, 730-737.

Boucher, J. C., Schurr, M. J. \& Deretic, V. (2000). Dual regulation of mucoidy in Pseudomonas aeruginosa and sigma factor antagonism. Mol Microbiol 36, 341-351.

Buck, M., Gallegos, M.-T., Studholme, D. J., Guo, Y. \& Gralla, J. D. (2000). The bacterial enhancer-dependent $\sigma^{54}\left(\sigma^{\mathrm{N}}\right)$ transcription factor. J Bacteriol 182, 4129-4136.

Datsenko, K. A. \& Wanner, B. L. (2000). One-step inactivation of chromosomal genes in Escherichia coli K12 using PCR products. Proc Natl Acad Sci U S A 97, 6640-6645.

Ernst, R. K., Guina, T. \& Miller, S. I. (2001). Salmonella typhimurium outer membrane remodelling: role in resistance to host innate immunity. Microbes Infect 3, 1327-1330.

Farewell, A., Brazas, R., Davie, E., Mason, J. \& Rothfield, L. I. (1991). Suppression of the abnormal phenotype of Salmonella typhimurium $\mathrm{rfaH}$ mutants by mutations in the genes for transcription termination factor Rho. J Bacteriol 173, 5188-5193.

Gunn, J. S. \& Miller, S. I. (1996). PhoP/PhoQ activates transcription of $p m r A / B$, encoding a two-component system involved in Salmonella typhimurium antimicrobial peptide resistance. J Bacteriol 178, 6857-6864.

Gunn, J. S., Lim, K. B., Krueger, J., Kim, K., Guo, L., Hackett, M. \& Miller, S. I. (1998). PmrA-PmrB-regulated genes necessary for 4aminoarabinose lipid A modification and polymixin resistance. Mol Microbiol 27, 1171-1182.

Guo, L., Lim, K. B., Gunn, J. S., Bainbridge, B., Darveau, R. P., Hackett, M. \& Miller, S. I. (1997). Regulation of lipid A modifications by Salmonella typhimurium virulence genes phoP-phoQ. Science 276, 250-253.

Hitchcock, P. J. \& Brown, T. M. (1983). Morphological heterogeneity among Salmonella lipopolysaccharide chemotypes in silver stained polyacrylamide gels. J Bacteriol 154, 269-277.

Hobbs, M. \& Reeves, P. R. (1994). The JUMPstart sequence: a 39 bp element common to several polysaccharide gene clusters. Mol Microbiol 12, 855-856. 
Ishimoto, K. S. \& Lory, S. (1989). Formation of pilin in Pseudomonas aeruginosa requires the alternative $\sigma$ factor (RpoN) subunit of RNA polymerase. Proc Natl Acad Sci USA 86, 1954-1957.

Joiner, K. A. (1988). Complement evasion by bacteria and parasites. Annu Rev Microbiol 42, 201-230.

Jones, B. D. \& Falkow, S. (1996). Salmonellosis: host immune responses and bacterial virulence determinants. Annu Rev Immunol 14, 533-561.

Karow, M., Raina, S., Georgopoulos, C. \& Fayet, O. (1991). Complex phenotypes of null mutations in the htr genes, whose products are essential for Escherichia coli growth at elevated temperatures. Res Microbiol 142, 289-294.

Klimpel, K. W., Lesley, S. A. \& Clark, V. L. (1989). Identification of subunits of gonococcal RNA polymerase by immunoblot analysis : evidence of multiple sigma factors. J Bacteriol 171, 3713-3718.

Klose, K. E. \& Mekalanos, J. J. (1998). Distinct roles of an alternative sigma factor during both free-swimming and colonizing phases of the Vibrio cholerae pathogenic cycle. Mol Microbiol 28, 501-520.

Koop, A. H., Hartley, M. E. \& Bourgeois, S. (1987). A low-copynumber vector utilizing $\beta$-galactosidase for the analysis of gene control elements. Gene 52, 245-256.

Kossack, R. E., Guerrant, R. L., Densen, P., Schadelin, J. \& Mandell, G. L. (1981). Diminished neutrophil oxidative metabolism after phagocytocis of virulent Salmonella typhi. Infect Immun 31, 674-678.

Leeds, J. A. \& Welch, R. A. (1996). RfaH enhances elongation of Escherichia coli hlyCABD mRNA. J Bacteriol 178, 1850-1857.

Leeds, J.A. \& Welch, R.A. (1997). Enhancing transcription through the Escherichia coli hemolysin operon, hlyCABD: RfaH and upstream JUMP-start DNA sequence function together via a postinitiation mechanism. J Bacteriol 179, 3519-3527.

Lesse, A. J., Campagnari, A. A., Bittner, W. E. \& Apicella, M. A. (1990). Increased resolution of lipopolysaccharides and lipooligosaccharides utilizing tricine-sodium dodecyl sulfatepolyacrylamide gel electrophoresis. J Immunol Methods 126, 109-117.

Lyczak, J. B., Zaidi, T. S., Grout, M., Bittner, W. M., Contreras, I. \& Pier, G. B. (2001). Epithelial cell contact-induced alterations in Salmonella enterica serovar Typhi lipopolysaccharide are critical for bacterial internalization. Cell Microbiol 3, 763-772.

Marolda, C. L. \& Valvano, M. A. (1998). Promoter region of the Escherichia coli O:7-specific lipopolysaccharide gene cluster: structural and functional characterization of an upstream untranslated mRNA sequence. J Bacteriol 180, 3070-3079.

Merrick, J. M. (1993). In a class of its own - the RNA polymerase sigma factor $\sigma^{\mathrm{N}}\left(\sigma^{54}\right)$. Mol Microbiol 10, 903-909.

Miller, J. H. (1972). Experiments in Molecular Genetics. Cold Spring Harbor, NY. Cold Spring Harbor Laboratory.

Nagy, G., Dobrindt, U., Kupfer, M., Emody, L., Karch, H. \& Hacker, J. (2001). Expression of hemin receptor molecule ChuA is influenced by $\mathrm{RfaH}$ in uropathogenic Escherichia coli 536. Infect Immun 69, 1924-1928.

Nieto, J. M., Bailey, J. A. M., Hughes, C. \& Koronakis, V. (1996). Suppression of transcription polarity in the Escherichia coli haemolysin operon by a short upstream element shared by polysaccharide and DNA transfer determinants. Mol Microbiol 19, 705-713.

Pang, T., Levine, M. M., Ivanoff, B., Wain, J. \& Finlay, B. B. (1998). Typhoid fever - important issues still remain. Trends Microbiol 6, 131-133.
Parkhill, J., Dougan, G., James, K. D. \& 38 other authors (2001). The complete genome sequence of a multidrug resistant Salmonella enterica serovar Typhi CT18. Nature 413, 848-852.

Pier, G., Grout, M., Zaidi, T., Meluleni, G., Mueschenborn, S. S., Banting, G., Ratcliff, R., Evans, M. J. \& Colledge, W. H. (1998). Salmonella typhi uses CFTR to enter intestinal epithelial cells. Nature 393, 79-82.

Pradel, E. \& Schnaitman, C. A. (1991). Effect of $r f a H(s f r B)$ and temperature on expression of $r f a$ genes of Escherichia coli K-12. J Bacteriol 173, 6428-6431.

Raetz, C. R. (1996). Bacterial lipopolysaccharides: a remarkable family of bioactive macroamphiphiles. In Escherichia coli and Salmonella typhimurium: Cellular and Molecular Biology, pp. 1035-1063. Edited by F. C. Neidhardt and others. Washington, DC: American Society for Microbiology.

Reeves, P. R. (1993). Evolution of Salmonella O antigen variation by interspecific gene transfer on a large scale. Trends Genet $\mathbf{9}$, $17-22$.

Rojas, G., Saldías, S., Bittner, M., Zaldívar, M. \& Contreras, I. (2001). The $r f a H$ gene, which affects lipopolysaccharide synthesis in Salmonella enterica Serovar Typhi, is differentially expressed during the bacterial growth phase. FEMS Microbiol Lett 204, 123-128.

Rosenberger, C. M., Scott, M. G., Gold, M. R., Hancock, R. E. W. \& Finlay, B. B. (2000). Salmonella typhimurium infection and lipopolysaccharide stimulation induce similar changes in macrophage gene expression. J Immunol 164, 5894-5904.

Schnaitman, C. A. \& Klena, J. D. (1993). Genetics of lipopolysaccharide biosynthesis in enteric bacteria. Microbiol Rev 57, 655-682.

Shingler, V. (1996). Signal sensing by sigma 54-dependent regulators: derepression as a control mechanism. Mol Microbiol 19, 409-416.

Sledjeski, D. D. \& Gottesman, S. (1996). Osmotic shock induction of capsule synthesis in Escherichia coli K-12. J Bacteriol 178, 1204-1206.

Stevens, M., Clarke, B. \& Roberts, I. (1997). Regulation of the Escherichia coli K5 capsule gene cluster by transcription antitermination. Mol Microbiol 24, 1001-1012.

Stout, V. \& Gottesman, S. (1990). RcsB and RcsC: a two component regulator of capsular synthesis in Escherichia coli. J Bacteriol 172, 659-669.

Takeda, S., Fujisawa, Y., Matsubara, M., Aiba, H. \& Mizuno, T. (2001). A novel feature of the multistep phosphorelay in Escherichia coli: a revised model of the $\mathrm{Rcs} C \rightarrow \mathrm{YojN} \rightarrow \mathrm{RcsB}$ signalling pathway implicated in capsular synthesis and swarming behaviour. Mol Microbiol 40, 440-450.

Tsai, C. M. \& Frasch, C.E. (1982). A sensitive silver stain for detecting lipopolysaccharide in polyacrylamide gels. Anal Biochem 119, 115-119.

Virlogeux, I., Waxin, H., Ecobichon, C., Lee, J. O. \& Popoff, M. Y. (1996). Characterization of the $r c s A$ and $r c s B$ genes from Salmonella typhi: rcsB through tviA is involved in regulation of Vi antigen synthesis. J Bacteriol 178, 1691-1698.

Wang, L. \& Gralla, J. D. (1998). Multiple in vivo roles for the -12-region elements of sigma 54 promoters. J Bacteriol 180, 5626-5631.

Wang, L. \& Reeves, P. R. (1994). Involvement of the galactosyl-1phosphate transferase encoded by the Salmonella enterica $\mathrm{rfbP}$ gene in O-antigen subunit processing. J Bacteriol 176, 4348-4356.

Wang, L., Liu, D. \& Reeves, P. R. (1996). C-terminal half of Salmonella enterica $\mathrm{WbaP}(\mathrm{RfbP})$ is the galactosyl-1-phosphate 
transferase domain catalyzing the first step of $\mathrm{O}$-antigen synthesis. J Bacteriol 178, 2598-2604.

Wang, L., Jensen, S., Hallman, R. \& Reeves, P. R. (1998). Expression of the $\mathrm{O}$ antigen gene cluster is regulated by $\mathrm{RfaH}$ through the JUMPstart sequence. FEMS Microbiol Lett 165, 201-206.

Wehland, M. \& Bernhard, F. (2000). The RcsAB box. Characterization of a new operator essential for the regulation of exopolysaccharide biosynthesis in enteric bacteria. J Biol Chem 275, 7013-7020.
Whitfield, C. \& Roberts, I. S. (1999). Structure, assembly and regulation of expression of capsules in Escherichia coli. Mol Microbiol 31, 1307-1319.

Zhao, H., Li, X., Johnson, D. E. \& Mobley, L. T. (1999). Identification of protease and $r p o N$-associated genes of uropathogenic Proteus mirabilis by negative selection in a mouse model of ascending urinary tract infection. Microbiology 145, 185-195.

Received 22 May 2002; revised 7 August 2002; accepted 20 August 2002. 